\section{Dilution of protein gel stain results in retention of staining capacity}

\author{
Rene C. Krieg ${ }^{1}$, Cloud P. Paweletz ${ }^{1}$, Lance A. Liotta ${ }^{1}$, and Emanuel F. \\ Petricoin III ${ }^{2}$ \\ ${ }^{1}$ National Institutes of Health, and ${ }^{2}$ Food and Drug Administration, \\ Bethesda, MD, USA
}

BioTechniques 35:376-378 (August 2003)
In 1975 , the invention of 2-D polyacrylamide gel electrophoresis (2-D PAGE) $(1,2)$ marked the beginning of what has now become a classic and dominant proteomics technology. Over the years, many refinements in the underlying technology have been made (reviewed in Reference 3), but some of the most important improvements and advancements have focused on increasing the sensitivity and visualization of the separated proteins. The techniques range from detection with scattered light following acetic acid fixation to highly sensitive silver staining techniques. In fact, there are many different silver staining techniques (4) that use more or less time-consuming protocols (5). As high-throughput and labor costs have become more and more important, the use of fluorescence dyes, following the simple "dump-and-wait-tostain" protocol, became popular.

One of the more popular commercially available staining reagents is SYPRO $^{\circledR}$ Ruby protein gel stain (Molecular Probes, Eugene, OR, USA) $(6,7)$. SYPRO Ruby is nearly as sensitive as silver staining (8), provides the advantage of a more linear correlation between protein amount and staining intensity, yields a dramatic improvement in ease of use over traditional silver stains, and, importantly, does not interfere with mass spectrometry-based protein identification methods (9). As an example, our high-sensitivity silver staining protocol requires at least $6 \mathrm{~h}$ with at least 14 changes of reagents, in comparison to the SYPRO Ruby protocol, which requires 2 changes of reagents with $30 \mathrm{~min}$ fixation and staining overnight.
However, as 2-D PAGE has become a routine practice in most laboratories now, and many investigators are employing either large-format type systems (e.g., Ettan ${ }^{\mathrm{TM}}$ DALT II system; 26 $\times 20 \mathrm{~cm}$; Amersham Biosciences, Piscataway, NJ, USA) or high-throughput smaller systems (Novex ${ }^{\circledR}$ Pre-Cast 7 $\mathrm{cm}$ Tris-Glycine; Invitrogen, Carlsbad, CA, USA), the amount of staining chemicals, and hence the cost required for any given experiment, has increased dramatically. Large-format gels can require up to $1500 \mathrm{~mL}$ staining solution/3 gels. The SYPRO Ruby protein gel stain-recommended staining protocol is to use the $1 \times$ stain as a ready-made solution. To evaluate whether we could reduce the cost associated with the SYPRO staining procedure without sacrificing staining sensitivity, we explored a series of stepwise dilutions of the reagent to determine the optimal concentration.

Dilution of SYPRO Ruby was performed in a qualitative (2-D gels) and a quantitative way (1-D gels) using a series of dilutions, with the $1 \times$ stock as the starting concentration and diluting quality water; Millipore, Bedford, MA, USA) of original purchased solution. Staining was performed overnight in the dark following a 30-min fixation in EtOH/acetic acid (50\%/10\% in Milli-Q water). Previous experiments in our laboratory indicated that a $1-\mathrm{h}$ rinsing in Milli-Q-quality water before scanning to enhance specificity and contrast was optimal. Figure 1 shows seven $2-\mathrm{D}$ gels $(\mathrm{PAA}, 9 \%-18 \%, \mathrm{pH}=$ 3-10) performed in parallel with the same amount of protein (lysate of SCCE-KYSE70 cells) loaded onto each gel with a variety of staining solutions. The comparison between silver and SYPRO Ruby staining shows nearly similar sensitivity as well as specificity in all cases. The relative number of observed spots as well as the ratio between low- and high-abundance proteins over the whole dilution range seems nearly unaltered using SYPRO stain at all evaluated concentrations. However, we would not recommend using a dilution of SYPRO Ruby to a lower extent than 1:5, as signal intensity decreases to levels where background staining becomes significant. This results in a poorer image quality even with increasing exposure times. Findings were reproducible over many replicates (data not shown). Interestingly, the 1:2 dilution delivered better results than the original SYPRO Ruby solution itself, every time.

A bovine serum albumin stock solution and a lysate of SCCE-KYSE70 cells were subsequently diluted and analyzed using 1-D PAGE (Novex PreCast Tris-Glycine 4\%-20\%) to evaluate the linearity of response to protein concentration. The gels were stained with SYPRO Ruby as described above, to a 1:20 concentration (in Milli- $\mathrm{Q}^{\circledR}$ -

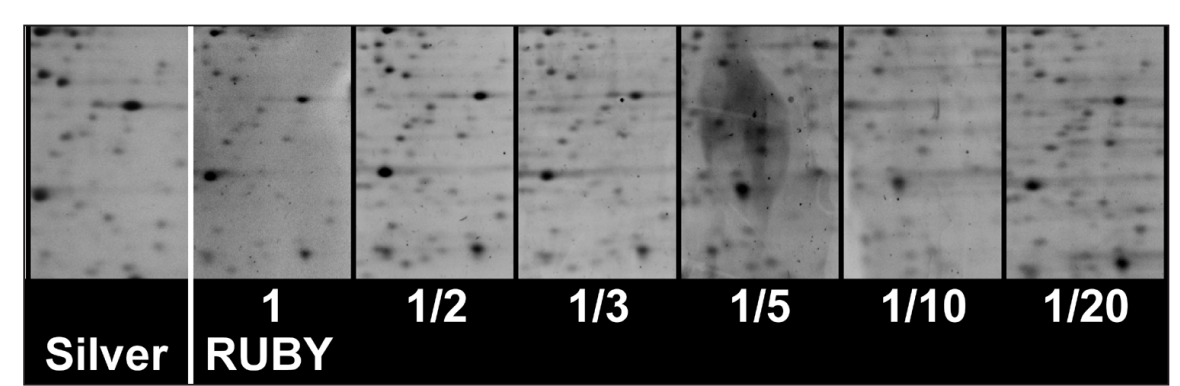

Figure 1. 2-D gels of SCCE-KYSE70 cell lysates. SYPRO Ruby staining in dilutions as indicated; corresponding silver staining (control) as indicated. 

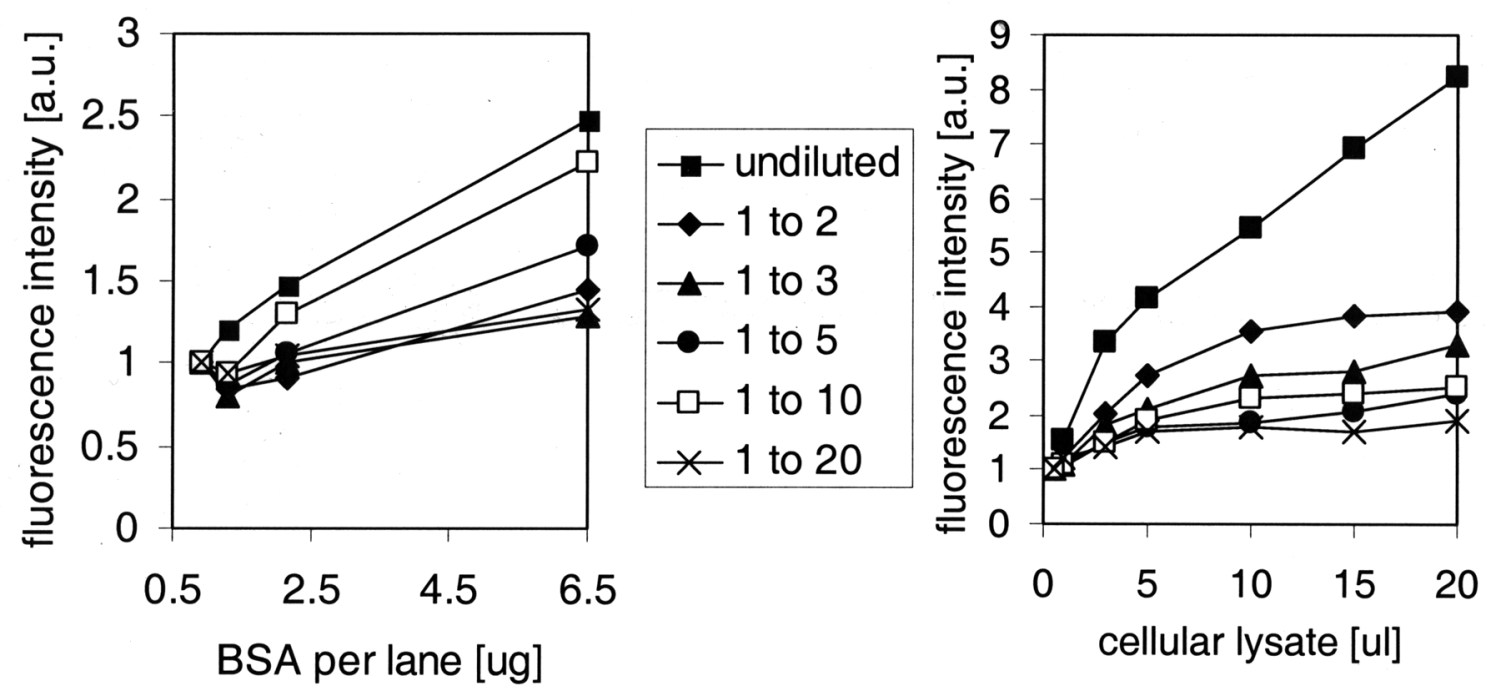

Figure 2. Normalized plots of 1-D gels after staining with SYPRO Ruby in dilutions as indicated. Fluorescence intensities in arbitrary units (a.u.) versus protein concentrations/lane in micrograms are plotted for bovine serum albumin stock solutions (left panel) and SCCE-KYSE70 lysates (right panel), respectively. Note the good linear correlation between protein concentration and intensity of fluorescence staining over the indicated protein concentration range as well as the very low detection limit demonstrated by bovine serum albumin $(1 \mu \mathrm{g})$.

and fluorescence intensities were scanned and quantified (FluorChem ${ }^{\mathrm{TM}}$; Alpha Innotech, San Leandro, CA, USA). Figure 2 shows the intensity of staining versus protein concentration in plot with all intensities normalized to the band of lowest concentration. Interestingly, all gels stained with diluted SYPRO Ruby showed overall comparable intensities. In addition, differences between the intensity ratios of the SYPRO Ruby 1:2 and 1:20 dilutions were less than the differences between the "original" SYPRO Ruby formulation and the $1: 2$ dilution.

In conclusion, for quantitative applications, we believe that the original stock $1 \times$ SYPRO Ruby concentration, as purchased from Molecular Probes, can be substantially diluted at least up to 1:5 for both 2-D and 1-D applications, reducing the cost dramatically while providing a reasonable alternative to time-consuming silver staining techniques. As with all dilution methods, however, a standard curve should be performed for the specific system being evaluated. It should also be noted that the dynamic range of the original SYPRO Ruby concentration is much higher than for any dilutions used.

\section{REFERENCES}

1.Klose, J. 1975. Protein mapping by combined isoelectric focusing and electrophoresis of mouse tissues. A novel approach to testing for induced point mutations in mammals. Humangenetik 26:231-243.

2.O'Farrel, P.H. 1975. High resolution two-dimensional electrophoresis of proteins. J. Biol. Chem. 250:4007-4021.

3.Rabilloud, T. 2002. Two-dimensional gel electrophoresis in proteomics: old, old fashioned, but it still climbs up the mountains. Proteomics 2:3-10.

4.Rabilloud, T. 1990. Mechanisms of protein silver staining in polyacrylamide gels: a 10 year synthesis. Electrophoresis 11:785-794.

5.Shevchenko, A., M. Wilm, O. Vorm, and M. Mann. 1996. Mass spectrometric sequencing of proteins silver-stained polyacrylamide gels. Anal. Chem. 68:850-858.

6.Gerner, C., S. Vejda, D. Gelbmann, E. Bayer, J. Gotzmann, R. Schulte-Hermann, and W. Mikulits. 2002. Concomitant determination of absolute values of cellular protein amounts, synthesis rates, and turnover rates by quantitative proteome profiling. Mol. Cell. Proteomics 1:528-537.

7.Korolainen, M.A., G. Goldsteins, I. Alafuzoff, J. Koistinaho, and T. Pirttila. 2002. Proteomic analysis of protein oxidation in Alzheimer's disease brain. Electrophoresis 23:3428-3433.

8.Nishihara, J.C. and K.M. Champion. 2002. Quantitative evaluation of proteins in one- and two-dimensional polyacrylamide gels using a fluorescent stain. Electrophoresis 23:22032215.

\begin{abstract}
9.Berggren, K., E. Chernokalskaya, T.H. Steinberg, C. Kemper, M.F. Lopez, Z. Diwu, R.P. Haugland, and W.F. Patton. 2000. Background-free, high sensitivity staining of proteins in one- and two-dimensional sodium dodecyl sulfate-polyacrylamide gels using a luminescent ruthenium complex. Electrophoresis 21:2509-2521.
\end{abstract}

Received 8 April 2003; accepted 21 May 2003.

Address correspondence to Rene C. Krieg, Clinical Proteomics Program, Laboratory of Pathology, National Cancer Institute, National Institutes of Health, Bethesda, MD 20892,USA.e-mail:krieg@cberfda.gov 